Original Article

\title{
Derivation of an empirical relation between the size of the nanoparticle and the potency of homeopathic medicines
}

\author{
Subrata Kar1,2, Poonam Bandyopadhyay,1,2, Sweta Chakraborty', Monalisa Chakrabarty ${ }^{1,2}$, \\ Biplab kumar Paul1,2 Sarbari Ghosh1,3, Ruma Basu1,4, Sukhen Das',2,5, Durga Sankar Bhar1, \\ Rajkumar Manchanda6, Anil Khurana ${ }^{6}$, Debadatta Nayak6, Papiya Nandy ${ }^{{ }^{*}}$
}

${ }^{1}$ Centre for Interdisciplinary Research and Education, Kolkata-700 068, India

${ }^{2}$ Physics Department, Jadavpur University, Kolkata- 700 032, India

3Vidyasagar Evening College, Kolkata, India

${ }^{4}$ Physics Department, Jogamaya Devi College, Kolkata 700 026, India

5Indian Institute of Engineering Science and Technology, Howrah 711103, India

${ }^{6}$ Central Council for Research in Homeopathy, New Delhi

*Corresponding author: pnandy00@gmail.com

\begin{abstract}
Homeopathic medicines are often prescribed at ultra-high dilutions and it is a clinically observed fact that the medicinal effect of the drug remains even at these high dilutions. The increase in potency of a medicine due to potentization is still debatable from physicochemical point of view. Out of various hypotheses to explain this phenomenon, a recent hypothesis, advanced by us and supported by others, is that the size of the constituent particles decreases and eventually achieves nano dimension due to potentization.

From the experiments performed by our group, the size of nanoparticles (NPs) of Cuprum metallicum, Zincum oxydatum, Aurum metallicum, Ferrum metallicum and Aconitum napellus $(6 \mathrm{cH}$, $30 \mathrm{cH}$ and $200 \mathrm{cH}$ ) has been estimated. A general mathematical expression of the form $y=a x^{\text {-n }}$ has been derived which relates the size of NPs (y) with the corresponding potencies (x).

There is no method to calculate the accurate potency of the homeopathic medicine, as the potency of a medicine depends to some extent on the method of preparation, for which a standardized procedure is warranted. Also, while handling a medicine, the solvent might evaporate causing a change in the potency. Thus by measuring the size of the NPs and using our proposed standard curve, the potency may be estimated.
\end{abstract}

Keywords: Nanoparticles, homeopathy, high dilution, size vs potency, nano dimension

\section{Introduction}

The fact that homeopathic medicines, propounded in 1796 by Samuel Hahnemann, are active at ultra-high dilutions (UHD) (even beyond Avogadro constant) and that effect of one medicine is different from another medicine of apparently zero concentration has thrown challenges to the scientific community at large. To prepare medicines of higher dilution, one $\mathrm{ml}$ of the mother tincture is diluted with $99 \mathrm{ml}$ of strong alcohol and given 10 "succussions" or "jerks" to prepare $1 \mathrm{cH}$ potency. In this way by successive dilution and succussions (together termed as potentization), homeopathic potencies are prepared. Few hypotheses have been

OPEN ACCESS

Cite as: Kar S, Bandyopadhyay P, Chakraborty S, Chakrabarty M, Paul BK, Ghosh S et al. Derivation of an empirical relation between the size of the nanoparticle and the potency of homeopathic medicines. Int J High Dilution Res. 2015;14(4):2-7. 
proposed to explain the biological action of UHD. ${ }^{1,2}$

A recent hypothesis, advanced by us and supported by others, proposed that due to potentization, the size of the constituent particles decreases and eventually reaches nano-dimension and a change in aspect ratio. ${ }^{3,4}$ We have verified the formation of nanoparticles (NPs) at higher dilutions by using field emission scanning electron microscope (FESEM), dynamic light scattering (DLS), transmission electron microscope (HRTEM) studies.5-9 ${ }^{5}$ The size of NPs measured by other research group ${ }^{10}$ vary, but the general trend is maintained.

The objective of the study is to form a general mathematical expression that relates size of NPs present in homeopathic medicines to its potency. Experimental evidence suggests that the potency and size of the NPs may be related to the biological effects though such correlations need rigorous verification.

Nandy et al have shown how a change in potency affects the membrane fluidity.3,5,9 Chakraborty et al have shown that change in potency of Cuprum metallicum causes inhibitory effect on the growth of gramnegative bacteria E.coli. ${ }^{11}$

Using the mean size of the NPs, as measured experimentally by us of five different medicines, viz., Cuprum metallicum ${ }^{5}$, Zincum oxydatum $^{6}$, Aurum metallicum ${ }^{7}$, Ferrum metallicum $^{8}$ and Aconitum napellus ${ }^{9}$, at three different potencies, $6 \mathrm{cH}, 30 \mathrm{cH}$ and $200 \mathrm{cH}$, we have fitted a geometric curve to this given set of bivariate data by least square method ${ }^{12}$.

\section{Methodology}

All the data presented here are the outcome of experiments performed by us. To determine the size of the NPs, we have used high-resolution transmission electron microscopy (HRTEM), FESEM and DLS, following the standard procedures as mentioned in the respective references. ${ }^{5-9}$ Briefly, for FESEM (Model FEI Quanta 250, USA) sample at different potencies $(6 \mathrm{cH}$, $30 \mathrm{cH}$ and $200 \mathrm{cH}$ ) was casted on a clean glass cover slip and was directly placed on carboncoated grid, sputter-coated with gold and then observed. DLS measurements of homeopathic samples were performed using a Nano-ZS 90-Malvern instrument (Model DLS-nano ZS, Zetasizer, Nano series, USA) employing a $4 \mathrm{~mW}$ He-Ne laser (l $1 / 4632.8$ $\mathrm{nm}$ ) equipped with a thermostatic sample chamber.

\section{Results and Discussions}

The particle sizes for all the five medicines at three potencies were measured several times. The average values of these measurements along with the standard deviation and the equation of the fitted curves are given in Table 1.

The observed sizes of the NPs are taken as the $\mathrm{y}$ variable and the potencies $6 \mathrm{cH}, 30 \mathrm{cH}$ and $200 \mathrm{cH}$ as $\mathrm{x}$ variable and are plotted as shown in the following diagrams: Cuprum metallicum (Fig. 1a), Zincum oxydatum ( Fig. 1b), Aurum metallicum (Fig. 1c), Ferrum metallicum (Fig. 1d) and Aconitum napelles (Fig. 1e).

The form of the equation $y=a x^{-n}$ is same for all medicines while the parameter values $(a$ and $n$ ) are specific for each medicine. The fit in each case is exceptionally good with nonlinear correlation coefficient, R-squared being very close to 1 in all cases. Here $R$ is the nonlinear correlation coefficient which

\section{OPEN}

Cite as: Kar S, Bandyopadhyay P, Chakraborty S, Chakrabarty M, Paul BK, Ghosh S et al. Derivation of an empirical relation between the size of the nanoparticle and the potency of homeopathic medicines. Int J High Dilution Res. 2015;14(4):2-7. 
indicates only how strongly the fitted curve is related to the observed one.

\begin{tabular}{|c|c|c|c|}
\hline Medicine & Potency (cH) & Mean size \pm SD $(\mathrm{nm})$ & Fitted equation and $R^{2}$ value \\
\hline \multirow[t]{3}{*}{ Cuprum metallicum } & 6 & $15.9 \pm 2.4$ & \multirow{3}{*}{$\begin{array}{l}y=61.01 x^{-0.91} \\
R^{2}=0.922\end{array}$} \\
\hline & 30 & $1.6 \pm 0.1$ & \\
\hline & 200 & $0.62 \pm 0.01$ & \\
\hline \multirow[t]{3}{*}{ Zincum oxydatum } & 6 & $83 \pm 52$ & \multirow{3}{*}{$\begin{array}{l}y=449.2 x^{-1.01} \\
R^{2}=0.985\end{array}$} \\
\hline & 30 & $11 \pm 1.3$ & \\
\hline & 200 & $2.3 \pm 0.6$ & \\
\hline \multirow[t]{3}{*}{ Aurum metallicum } & 6 & $345 \pm 50$ & \multirow{3}{*}{$\begin{array}{l}y=764.5 x^{-0.51} \\
R^{2}=0.95\end{array}$} \\
\hline & 30 & $104 \pm 30$ & \\
\hline & 200 & $55 \pm 12$ & \\
\hline \multirow[t]{3}{*}{ Ferrum metallicum } & 6 & $167 \pm 43$ & \multirow{3}{*}{$\begin{array}{l}y=341.4 x^{-0.39} \\
R^{2}=0.999\end{array}$} \\
\hline & 30 & $92 \pm 37$ & \\
\hline & 200 & $42 \pm 2$ & \\
\hline \multirow[t]{3}{*}{ Aconitum napellus } & 6 & $135 \pm 15$ & \multirow{3}{*}{$\begin{array}{l}y=333.2 x^{-0.51} \\
R^{2}=0.999\end{array}$} \\
\hline & 30 & $58 \pm 7$ & \\
\hline & 200 & $23 \pm 3$ & \\
\hline
\end{tabular}

Table 1: Experimental values of average particle sizes against potency of five different homeopathic medicines and the corresponding fitted equations.

Comparison of our result ${ }^{8}$ with that reported remains the same. There might be some by Rajendran ${ }^{10}$ shows a difference in the values of the size of the particle of Ferrum underlying reason for this observation which metallicum, though the general trend i.e., attaining nano-dimension at higher potency
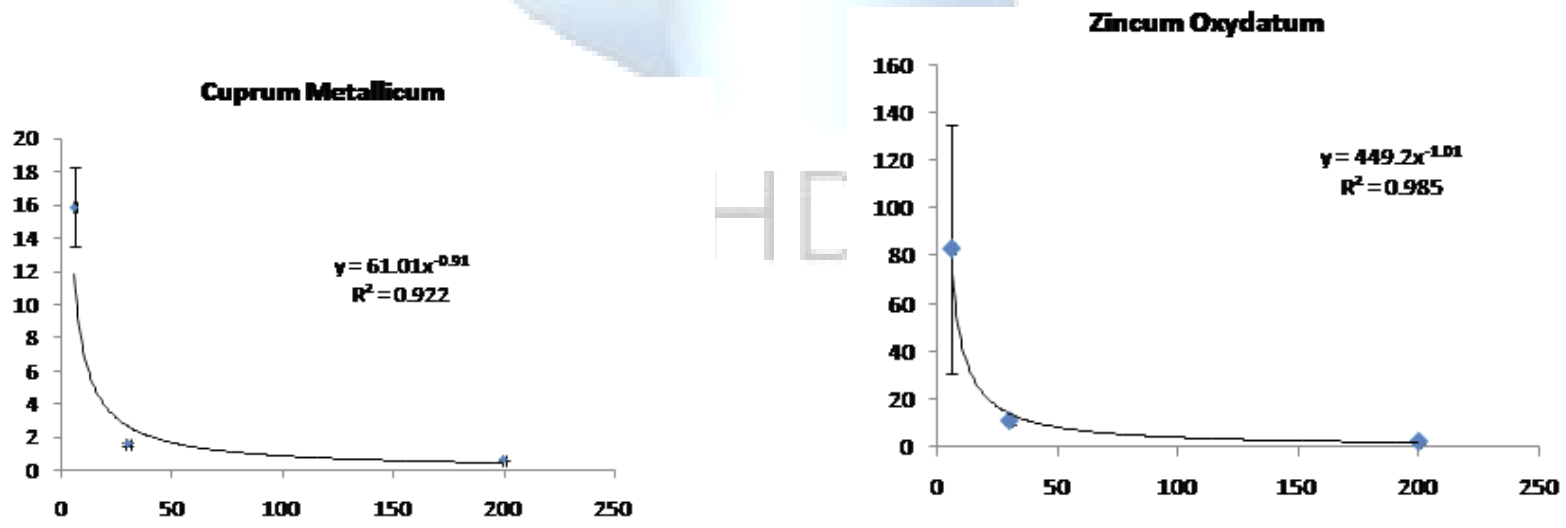

OPEN ACCESS

Cite as: Kar S, Bandyopadhyay P, Chakraborty S, Chakrabarty M, Paul BK, Ghosh S et al. Derivation of an empirical relation between the size of the nanoparticle and the potency of homeopathic medicines. Int J High Dilution Res. 2015;14(4):2-7. 

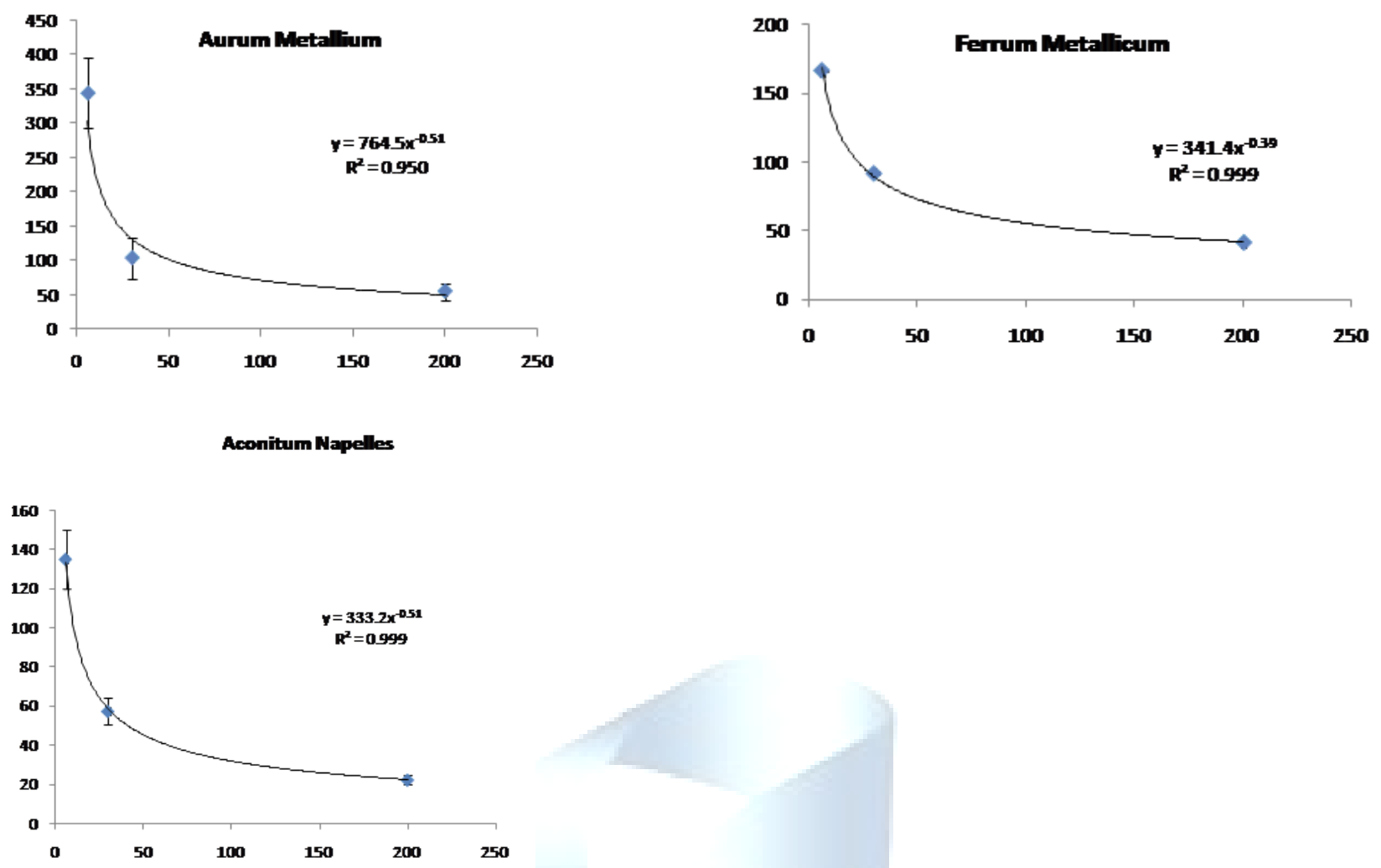

Figure 1 Experimental values of the size and potencies for a) Cuprum metallicum, b) Zincum oxydatum, c) Aurum metallicum, d) Ferrum metallicum and e) Aconitum napelles; $\mathrm{x}$ axis=potency, y axis=size of nanoparticles.

The nature of the curves is found to remain the same for all the medicines, but the parameters $a$ and $\mathrm{n}$ vary for different medicines, making the fitted curve characteristic of respective medicine. Hence for a particular potency, the size of the constituent NPs can be estimated by referring to the respective curve. The study proposes that the size of the particles of a medicine differs from that of another at the same potency and it decreases as the dilution increases.

The values of $\mathrm{R}^{2}$ for all the five medicines show that the fitted geometric curves approximate the experimental ones very closely.

\section{Conclusion}

Our earlier works have confirmed that the size of the NPs reduces with increase in potency.5-9 Here we have summed up the results obtained with different medicines at different potencies and have been able to derive a general mathematical expression of the form $y=a x^{-n}$.

The present study proposes that

a) The size of the constituent particles of a homeopathic medicine bears a definite polynomial relation with the potency of the medicine, without implying any casualization. It is a

\section{OPEN ACCESS}

Cite as: Kar S, Bandyopadhyay P, Chakraborty S, Chakrabarty M, Paul BK, Ghosh S et al. Derivation of an empirical relation between the size of the nanoparticle and the potency of homeopathic medicines. Int J High Dilution Res. 2015;14(4):2-7. 
predictive relationship and statistical dependence is not sufficient to indicate a causal relationship. The knowledge of the potency of the medicine will give an estimate of the size of the constituent particles.

b) Each homeopathic medicine has a characteristic curve for size versus potency.

c) The knowledge of the potency of the medicine will give an estimate of the size of the constituent particles.

\section{Conflict of Interest}

The authors report no conflict of interest.

\section{Financial statement}

The work was done with the financial support from a collaborative project with Central Council for Research in Homeopathy (CCRH), Under Ministry of AYUSH, Govt. of India (F. No. 6-01/14-15/CCRH/cash/11515)

\section{Acknowledgement}

The work has been done in technical collaboration with the CCRH. Stimulating discussion with Dr. A. Nandy is thankfully acknowledged.

\section{References}

1. Anick DI, Ives IA. The silica hypothesis for homeopathy: physical chemistry. Homeopathy 2007; 96: 189-95.

2. Elia V, Napoli E, Germano R. The 'memory of water': an almost deciphered enigma. Dissipative structures in extremely diluted aqueous solutions of the homeopathic medicine. Homeopathy 2007;96(3): 163-69.
3. Nandy P, Bhandary S, Das S, Basu R and Bhattacharyya S. Nanoparticles and membrane anisotropy. Homeopathy 2011;100(3): 194.

4. Chikramane PS, Kalita D, Suresh AK, Kane SG, Bellare IR. Why extreme dilutions reach non-zero asymptotes: A nanoparticulate hypothesis based on froth dilution. Langmuir 2012;28: 15864-75.

5. Ghosh S, Chakrabarty M, Das, S, Basu, R, Nandy P. Effect of different potencies of nanomedicine Cuprum metallicum on membrane fluidity - a biophysical study. Am. I. Hom. Med. 2014; 107(4): 161-69.

6. Bandyopadhyay P, Nandy P, Basu, R, Dhar DS, Das, S. Effect of dilution on thermovoltage generation using homeopathic nanomedicine Zincum oxydatum. International Journal of Innovative Research in Science Engineering and Technology 2015; 3(6): 226-30.

7. Kar S, Chakrabarty M, Nandy P, Basu, R. Characterization and Hemocompatibility of Aurum metallicum for its potential use as therapeutic injectables. Complementary Therapies in Medicine (Under review)

8. Chatterjee A, Paul BK, Gayen A, Das S, Nandy P. Effect of potency of homeopathic Nanomedicines on electrical properties of thin film of PVDF-HFP. Proceedings International conference on advances in Bioprocess Engineering and Technology 2016. (accepted for presentation)

9. Chakrabarty M, Ghosh S, Das S, Basu R, Nandy P. Effect of different potencies

OPEN ACCESS

Cite as: Kar S, Bandyopadhyay P, Chakraborty S, Chakrabarty M, Paul BK, Ghosh S et al. Derivation of an empirical relation between the size of the nanoparticle and the potency of homeopathic medicines. Int J High Dilution Res. 2015;14(4):2-7. 
of nanomedicine Aconitum napellus on its spectral and antibacterial

properties. International Journal of Innovative Research in Science Engineering and Technology 2015; 3: 6861-67.

10. Rajendran ES. An evaluation of Avogadro's number in the light of HRTEM and EDS studies of high dilutions of Ferrum metallicum 6, 30, 200,1M,10M and 50Mc. Int J of High Dilution Res. 2015; 14(3):3-9.
11. Chakraborty M, Das S, Manchanda RK, Basu R, Nandy P. Application of nanomedicine Cuprum metallicum as an agent for remediation of an azo dye, methyl orange and study its associated antimicrobial activity. Int. J. Environmental Sciences 2015 (In press).

12. Goon, Gupta and Dasgupta: Fundamentals of Statistics, Vol 1, The World Press Private Limited, 1975.

Received: August 24, 2015. Accepted: December 02, 2015.

(C) International Journal of High Dilution Research.

Not for commercial purposes.
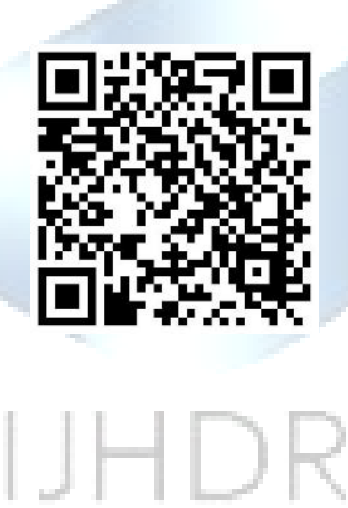